\title{
Development of Technology for Generating Stimulus Temperature Sensation with Skin Temperature Measurement Function
}

\author{
Koshiro Honda a , Yoshihumi Oyama ${ }^{\text {b, }}$,Masanobu Nagata ${ }^{\mathrm{b}}$, Miki Oogushi ${ }^{\mathrm{c}}$, Mika Hagino ${ }^{\mathrm{c}}$ \\ ${ }^{a}$ Advanced Course of Electronics and Information Systems Engineering Kumamoto NCT \\ 2659-2, Suya Koshi, Kumamoto, Japan \\ ${ }^{b}$ Kumamoto National College of Technology, 2659-2, Suya Koshi, Kumamoto, Japan \\ 'Kumamoto Univaersity Hospital, 1-1-1, Honjo Kumamoto, Kumamoto, Japan \\ *Yoshifumi Oyama : oyama@ kumamoto-nct.ac.jp
}

\begin{abstract}
The complex inspection device is composed of a controller and four sensibility inspection devices for pain, thermal, tactile, and vibratory sensation. This paper describes TSID (Thermal Sensibility Inspection Device). We add a new skin temperature measurement function in this TSID. We experimented actually to measure the temperature of human skin using TSID of introducing the test formula for calculating a skin temperature in consideration of the outside air temperature. As a result of comparison of the temperature of the skin by a thermometer and TSID, was possible to fit the $2.0^{\circ} \mathrm{C}$ less error. It is considered from the experimental results, it was able to be added to the TSID a function to measure the skin temperature.
\end{abstract}

Keywords: Quantitative, Complex inspection, Cutaneous Sense, Peltier element;

\section{Introduction}

Nurses and physical therapists in the hospital, when inspecting patient's cutaneous sensation, use the various implements which respond to the type of sense. For example, they use a needle for pain inspection, a test tube for thermal sensibility inspection, and a piece of cotton for tactile inspection. These inspection methods are troublesome and inspection time is long. Furthermore, these inspections are not quantitative, and these results are affected by only the subjectivity of medical workers. As for quantitative inspection, the electronic devices are marketed generally. But, because these devices are large scale and these are hard to treat, they are not often used. They are effective on the precise inspection, but not effective on the screening inspection. Therefore, to shorten patient's waiting time, to reduce medical worker's load, and to establish a quantitative diagnosis of cutaneous sense, it is necessary for medical workers to inspect simply, efficiency, and speedy ${ }^{(1-2)}$. In laboratory, we are developing to the complex inspection device. It is composed of a controller and four sensibility inspection devices for pain, thermal, tactile, and vibratory sensation. Figure 1 shows the controller of this complex inspection device. This paper describes TSID (Thermal Sensibility Inspection Device). Figure 2 shows the TSID prove. TSID is keeps temperature by controlling the amount of current flows peltier element by PID control using IC driver. In that step through the experiment, if the skin temperature measuring function to the TSID, it has been found that it is possible to stimulate the subject at an accurate temperature. To clarify the performance of its functions, add a new skin temperature measurement function in this TSID.

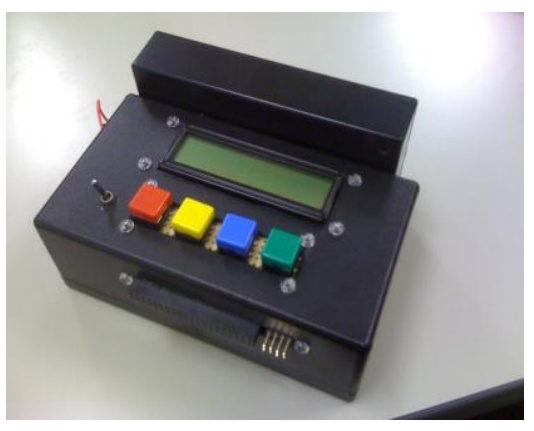

Fig. 1. Complex Inspection Device for Cutaneous Sensing Diagnosis. 


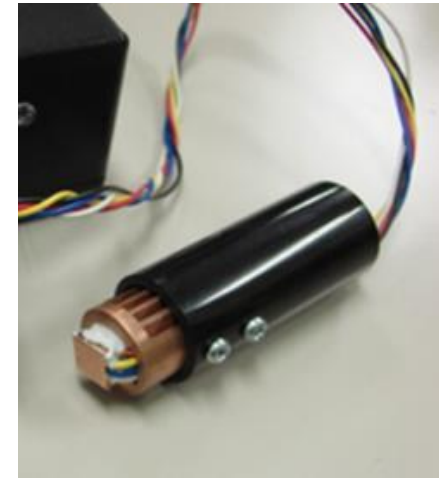

Fig.2. The picture of TSID prove.

\section{TSID}

In the sensory examination, there are temperature sense inspection, vibratory sense inspection, tactile sense inspection, and tenderness objective sense inspection. In these inspections, we have a research of equipment development about the temperature sense inspection, the vibratory sense inspection, and the tactile sense inspection. To do these inspections in one controller, we are developing a removable device each stimulus probe at cartridge type. The controller is equipped with a 8-bit microcontroller for generating a stimulation signal in the PWM control. Figure 1 shows a control box body of the test apparatus. Its size is $130 \times 80 \times 40 \mathrm{~mm}$. TSID has the function of setting a temperature of the contact surface at intervals of $5^{\circ} \mathrm{C}$ from $5^{\circ} \mathrm{C}$ to $45^{\circ} \mathrm{C}$. Table 1 shows the specification of the TSID. Figure 3 shows the structure of TSID prove. It is composed of a copper plate, a temperature sensor a copper connector, a peltier element, and a heat sink, in order from the contact surface.

\subsection{PMW control}

PWM controller is used to control voltage values that apply to the peltier element. PWM controller determines the voltage value by the pulse width of voltage. When the power supply voltage is $\mathrm{V}[\mathrm{V}]$, the output voltage Vo is expressed by Eq.(1). Here, D is [ pulse width / period ] in Figure 4.

$$
V o=V \times D
$$

Tablel.1. Specification of Sense of temperature stimulus probe.

\begin{tabular}{|c|c|}
\hline Item & Specifications \\
\hline $\begin{array}{c}\text { Setting } \\
\text { temperature }\end{array}$ & $\begin{array}{c}5,10,15,20,25,30,35,40,45,50^{\circ} \mathrm{C} \\
\text { Max. temp. } 55^{\circ} \mathrm{C}, \text { Min. temp } 5^{\circ} \mathrm{C}\end{array}$ \\
\hline Contact area & $9 \times 9 \mathrm{~mm}$ \\
\hline Weight & $49 \mathrm{~g}$ \\
\hline Size & Length:80mm Diameter; $10 \mathrm{~mm}$ \\
\hline
\end{tabular}

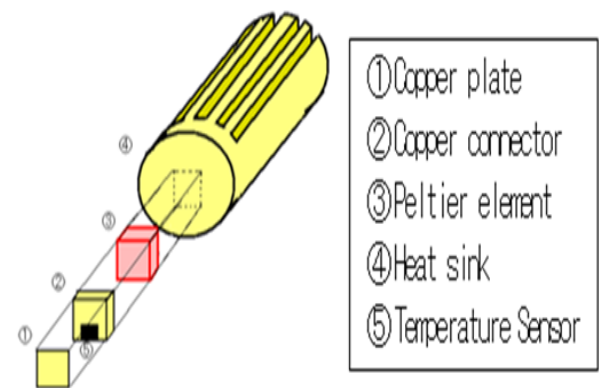

Fig. 3. Structure of TSID prove.

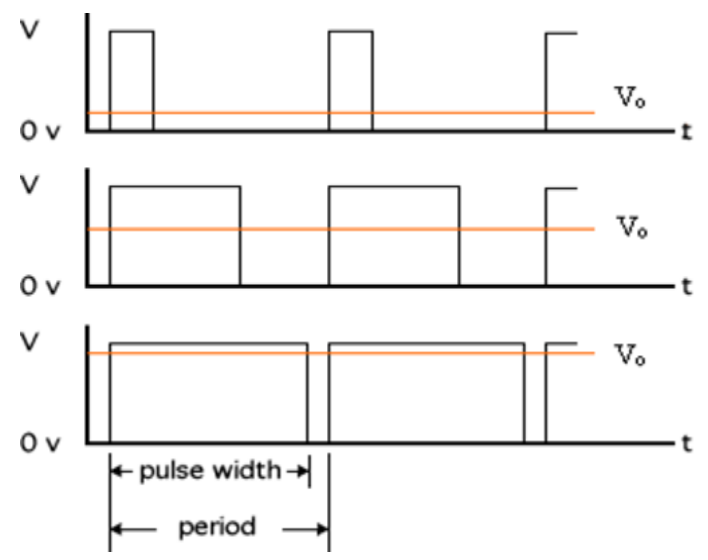

Fig. 4. PWM controller.

\subsection{PID control}

TSID controls the electrical current value through the peltier element by PID controller to make constant temperature on the contact surface. PID controller determines pulse width of voltage.

$$
P=k_{1} e_{1}+k_{2} s+k_{3}\left(e_{1}-e_{2}\right)
$$

$e_{1}:$ The temperature deviation

$e_{2}:$ Last temperature deviation

$\mathrm{s}:$ The sum of deviations

$k_{1}, k_{2}, k_{3}$ : Arbitrary constant 
The proportional term 'e1' equals the subtracting value of contact surface temperature from the preset temperature. The integral term's' prevents temperature's fluctuations in the vicinity of the preset temperature. The derivation term '(e1-e2)' prevents the momently changing of temperature ${ }^{(3-}$ 4).

\section{EXPERIMENT OF TSID PERFORMANCE}

\subsection{Performance evaluation experiment}

We experimented for evaluating the performance of TSID. We used a thermometer [AM-2001] as standard measuring instrument. Figure 5 is the result that the preset temperature is $45^{\circ} \mathrm{C}$. Figure 6 is the result of case of $5^{\circ} \mathrm{C}$. Figure 5 and Figure 6 show that TSID' s temperature is equal to the temperature measured using the AM-2001. It takes 6 seconds for increase the temperature to $45^{\circ} \mathrm{C}$ from the initial temperature, although it takes 15 seconds for decrease temperature to $5^{\circ} \mathrm{C}$ from the initial temperature. The reason of taking more time to cool is due to the Joule heat generated by the electrical current flowing through the peltier.

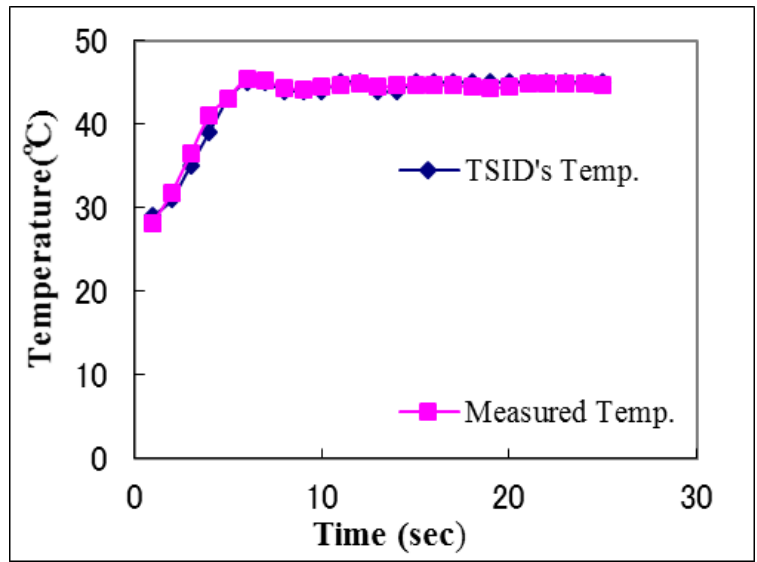

Fig. 5. Case of $45^{\circ} \mathrm{C}$.

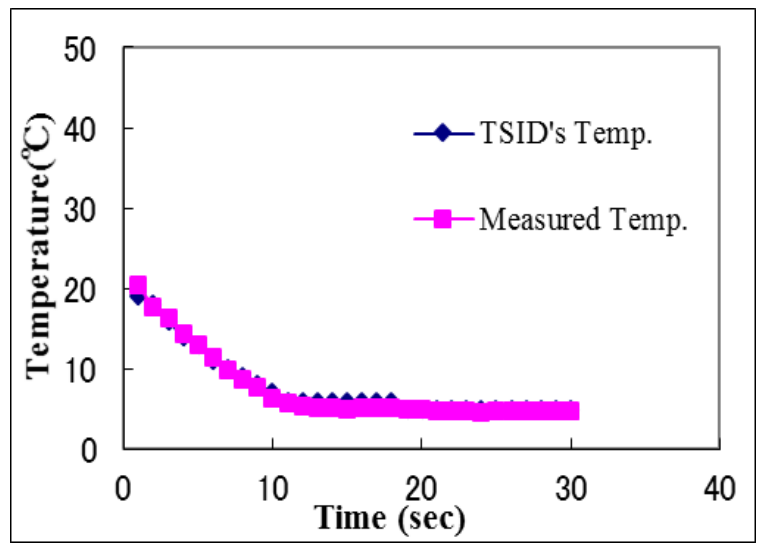

Fig. 6. Case of $5^{\circ} \mathrm{C}$.

\subsection{Sensing properties experiment}

We tested how persons feel the temperature of TSID. The values of sensory evaluation were five steps shown in Table 2. According to the medical institution, seems to be if there is a test item of five stages. Subjects were 23 healthy students. Figure 7 shows the result of this experiment. The vertical axis shows each value of sensory evaluation, and the horizontal axis shows each value of preset temperature. In the case of $15^{\circ} \mathrm{C}$, many subjects feel cold.

Table. 2. Evaluation value of temperature sensation.

\begin{tabular}{|c|c|}
\hline value & sensory step \\
\hline 1 & Cold \\
\hline 2 & Cold a little \\
\hline 3 & Nothing feel \\
\hline 4 & Hot a little \\
\hline 5 & Hot \\
\hline
\end{tabular}

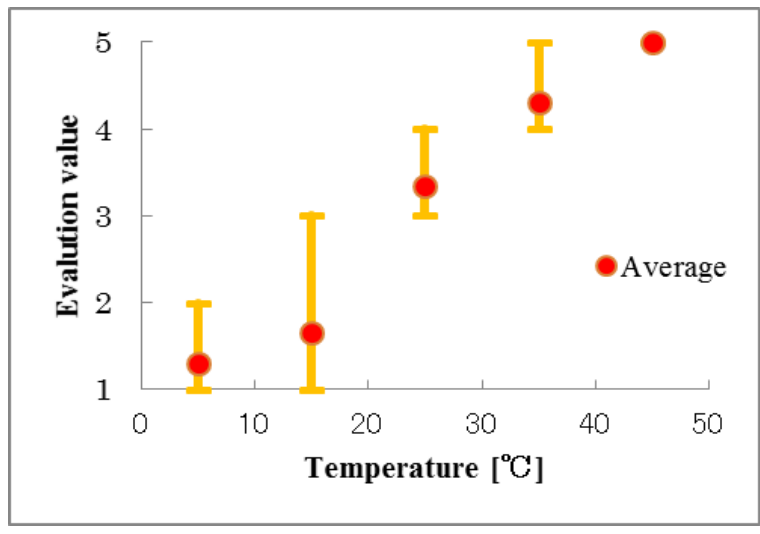

Fig. 7. Evaluation of the TSID's temperature. 


\section{SKIN TEMPERATURE MEASUREMENT FUNCTION}

\subsection{Configuration of TSID}

Figure 8 shows a configuration of each device of the TSID. When measuring the temperature of the copper plate that contacts the skin, the temperature sensor, since the simultaneously sensed ambient temperature and the temperature of the contact surface, it is not possible to measure accurately only the contact temperature. To solve this problem, we have developed a method, to derive a test formula for estimating the temperature of the contact surfaces in consideration of the ambient temperature, measuring the temperature of the contact surface of the test formula. A known heat source was touched the contact surface for 3 seconds, after that we measured the temperature variation of the sensor between the 3 seconds. Ambient temperature at this time is $25^{\circ} \mathrm{C}$ and $20^{\circ} \mathrm{C}$. Figure 9 and Figure 10 show the approximate curve equation the measurement result at this time.

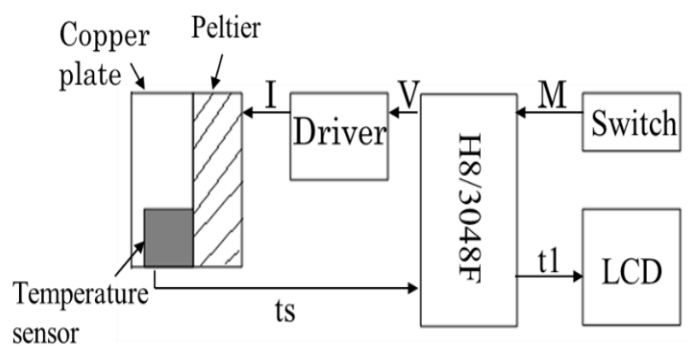

Fig. 8. Configuration of TSID.

(I:current, V:voltage, M:manual operation, ts:Temperature sensing, $\mathrm{t} 1$ :temperature of the contact surface)

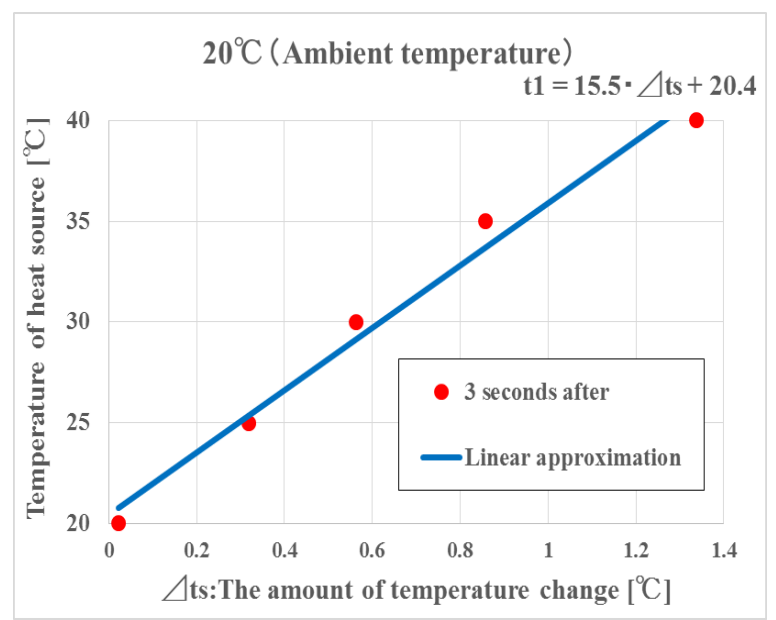

Fig. 9. Case of $20^{\circ} \mathrm{C}$.

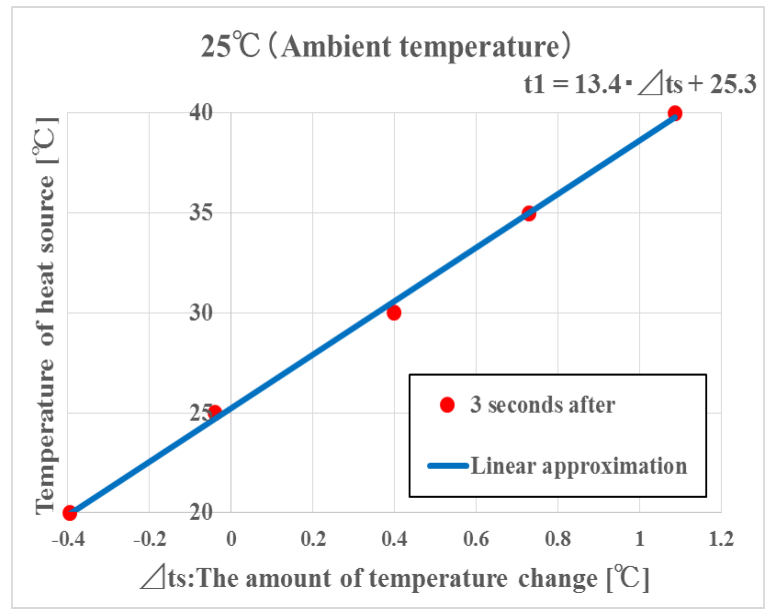

Fig. 10. Case of $25^{\circ} \mathrm{C}$.

Test formula for estimating the contact temperature from the linear approximation equation obtained from this experiment were derived as follows.

$$
t_{1}=\alpha \cdot \Delta t_{s}+\beta
$$

( $\alpha, \beta$ : parameters to be determined by experiment)

The value of $\alpha$ and $\beta$ vary with the ambient temperature which measures. For example, in the case of outdoor temperature $20{ }^{\circ} \mathrm{C}, \quad \alpha=15.5$ and $\beta=20.4$.

\subsection{Skin temperature measurement experiment}

We programmed derived test expression, and incorporated in the microcomputer of the controller. We measured the temperature of each portion of subjects using this TSID prove. Each measurement parts were at the back of the hand, palm, arm, cheek, and instep of the foot, and, those are easy to measure. The thermometer (HD-1200K) was used as the reference for measuring the temperature. Measured temperature of TSID probe was compared with measured temperature of thermometer. Figure 11 shows the measurement result of the back of the hand, Figure 12 shows of palm, Figure 13 shows of arm, Figure 14 shows of cheek, and, Figure 15 shows of instep of the foot. 


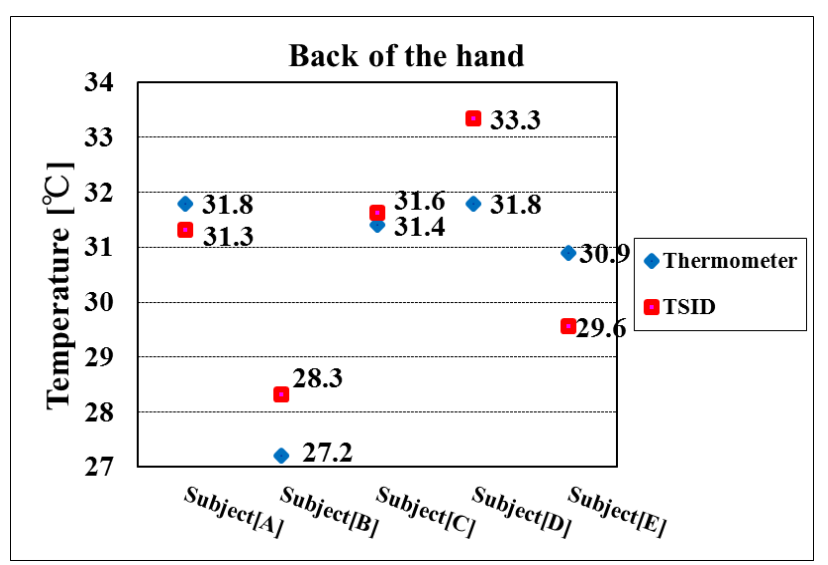

Fig. 11. Case of back of the hand.

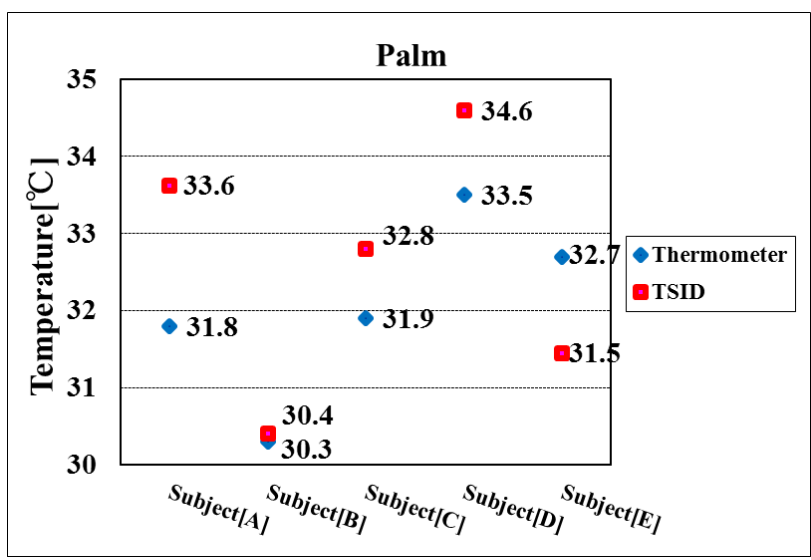

Fig. 12. Case of Palm.

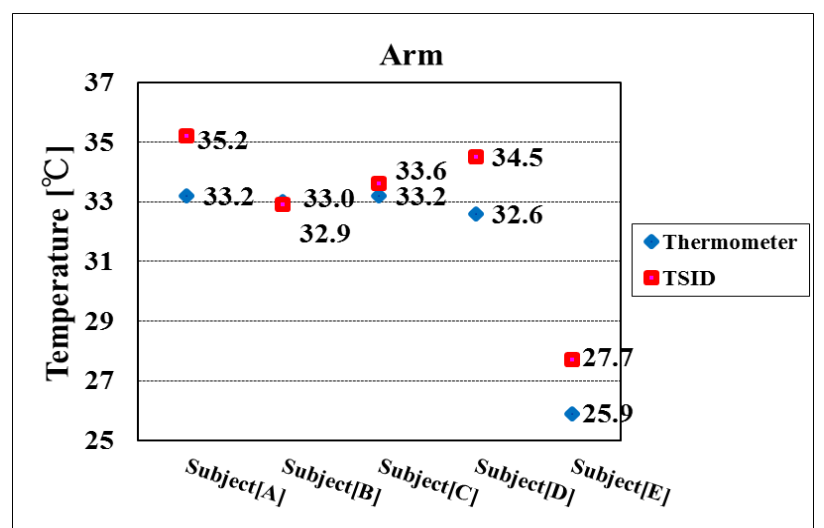

Fig. 13. Case of Arm.

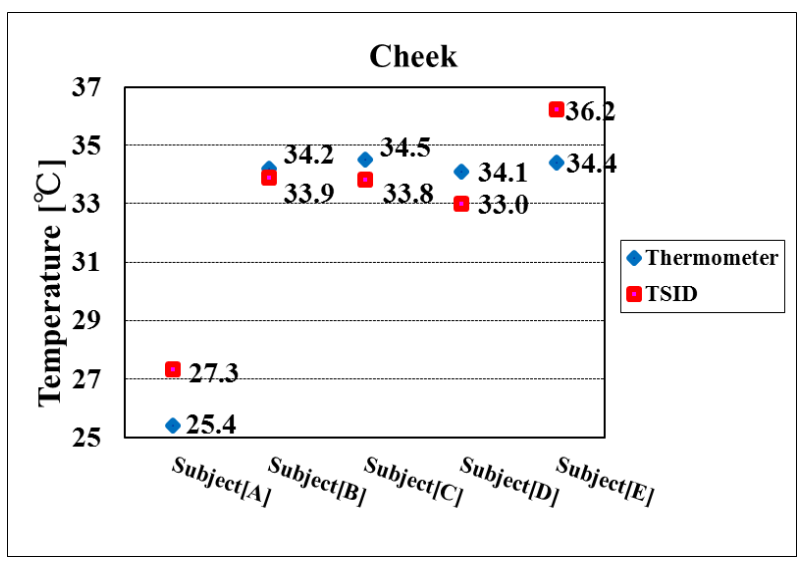

Fig. 14. Case of Cheek.

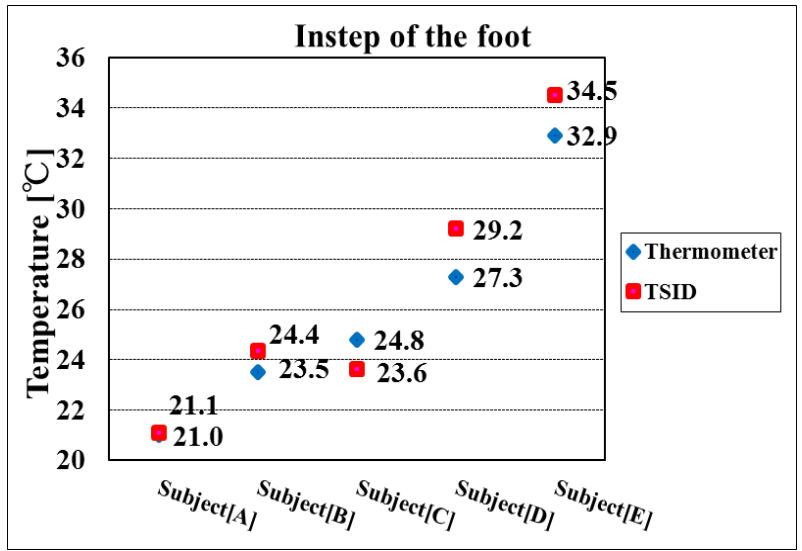

Fig. 15. Case of instep of the foot.

The measurement errors of TSID probe for measurements of HD- $1200 \mathrm{~K}$ is within $2^{\circ} \mathrm{C}$. From this result, we have determined that it is possible TSID probe has a temperature measuring function. However, to be suppressed error within $1{ }^{\circ} \mathrm{C}$ is a future challenge. As a further issue, it TSID takes about 10 minutes to acclimate to ambient temperature, and when the skin temperature and the ambient air temperature is close, there is a problem of error in the temperature measurement value increases.

\section{Conclusions}

We showed the performance of the TSID by the experiment. Further, it was possible to add a function capable of measuring the skin temperature TSID. We are testing to evaluate the performance of the TSID with the medical staffs. And we are studying on the inspection devices for tactile, and vibration sensation ${ }^{(5)}$. 


\section{Acknowledgment}

We would you like to thank people for cooperate with the experiment and the help received.

\section{References}

(1) Y.Oyama and T.Tokunaga, "Development of Complex Inspection Device for Cutaneous Sense Diagnosis", in Qingdao, ICIC-2007-IS19-004, September 2007.

(2) D.Tokunaga, "Development of Complex Inspection Device for Cutaneous Sense Diagnosis", $16^{\text {th }}$ Kosen Symposium proceedings pp.37, Febrary 2011.

(3) T.Maki, "Performance evalution of the thermesthesia stimulus probe in Complex Inspection Device for Cutaneous Sense Diagnosis", The $65^{\text {th }}$ Joint Conference of Electrical and Electronics Engineers in Kyusyu 071P-08, September 2012.

(4) Y.Oyama and M.Nagata and M.Ogushi and M.Hagino, "Composite type inspection machine development for quantitative sensory testing in medical institutions", The Institute of Industrial Applications Engineers S3-1, September 2014.

(5) K.Hamasaki, "Performance evalution of the Vibratory sensibility stimulus probe in Complex Inspection Device for Cutaneous Sense Diagnosis", The $65^{\text {th }}$ Joint Conference of Electrical and Electronics Engineers in Kyusyu 07-1P-06, September 2012. 